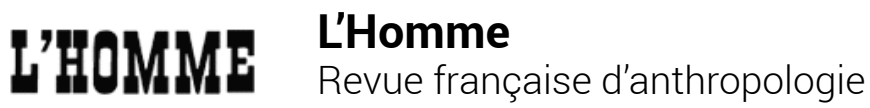

\section{9-220 | 2016}

Pacifications urbaines

\section{After the Ancestors. An Anthropologist's Story}

, Cambridge, Cambridge University Press, 2015, 372 p., index, ill.

\section{Bernard Formoso}

\section{(2) OpenEdition}

Journals

Édition électronique

URL : http://journals.openedition.org/lhomme/29171

DOI : 10.4000//homme.29171

ISSN : 1953-8103

Éditeur

Éditions de l'EHESS

\section{Édition imprimée}

Date de publication : 29 novembre 2016

ISSN : 0439-4216

\section{Référence électronique}

Bernard Formoso, «After the Ancestors. An Anthropologist's Story », L'Homme [En ligne], 219-220 |

2016, mis en ligne le 28 novembre 2018, consulté le 23 septembre 2020. URL : http://

journals.openedition.org/lhomme/29171; DOI : https://doi.org/10.4000/lhomme.29171

Ce document a été généré automatiquement le 23 septembre 2020.

() École des hautes études en sciences sociales 


\title{
After the Ancestors. An Anthropologist's Story
}

, Cambridge, Cambridge University Press, 2015, 372 p., index, ill.

\author{
Bernard Formoso
}

\section{Andrew Beatty, After the Ancestors. An Anthropologist's Story, Cambridge, Cambridge University Press, 2015, 372 p., index, ill.}

1 LE TITRE de l'ouvrage - After the Ancestors - est volontairement ambigu. Il exprime une rupture s'il est traduit par "Après les ancêtres ", tandis qu'il suggère un certain degré de continuité culturelle portée par l'idéologie de la filiation lorsqu'il est rendu par « À la suite des ancêtres ». Or, c'est précisément d'une rupture dans la continuité dont Andrew Beatty restitue les aspects les plus saillants dans l'étude des habitants de l'île de Nias - les Niha -, dont il a partagé le quotidien de 1986 à 1988. Ce livre répond à une double ambition : rendre le plus fidèlement possible la manière dont évoluèrent dans le temps les rapports de l'ethnographe avec ses sujets d'étude; utiliser cette expérience inter-subjective et les conflits dont l'auteur fut le témoin comme autant de révélateurs des dilemmes religieux et des tensions sociales que cette société locale a vécus, à la suite des profonds changements provoqués par la colonisation, la modernisation et la conversion religieuse.

2 Selon l'auteur, la rupture du lien avec les ancêtres qui médiatisaient jusqu'alors le rapport à la surnature remonte aux années 1910. Durant cette décennie, les colonisateurs hollandais mirent fin aux chefferies, à l'esclavage et à la pratique de la chasse aux têtes chez les Niha, tandis que des pasteurs luthériens développaient sur place leurs œuvres missionnaires. D'abord d'ampleur modeste, la conversion au christianisme se généralisa au cours des années 1920, dans le cadre d'un "grand repentir » orchestré par les prêtres occidentaux, ceux-ci assimilant les épidémies de variole qui décimaient la population à une sanction divine touchant tous ceux qui se tenaient à l'écart de la foi. Si les Niha détruisirent alors les mégalithes et autres icônes de leurs anciens cultes pour se tourner vers le Tout-Puissant chrétien, opportunément 
assimilé par les traducteurs de la Bible au dieu démiurge de leur mythologie, ils n'en effacèrent pas pour autant de leur imaginaire les ancêtres, dont le pouvoir de sanction reste aujourd'hui encore redouté. Cette résilience des figures de l'ancestralité tient pour partie à l'organisation de la société en clans exogames dont l'ancrage territorial, la profondeur généalogique et l'unité cultuelle d'antan sont matérialisés par de "grandes maisons " soigneusement restaurées, même si beaucoup sont désertées du fait même qu'elles sont saturées de présence ancestrale. Elle s'explique aussi par une économie du prestige qui conditionne l'accès aux fonctions du pouvoir et qui, comme jadis les offrandes adressées aux ancêtres, repose sur le sacrifice de porcs, quand bien même cette pratique "païenne » est réprouvée par l'Église. Enfin, remarque Andrew Beatty, la protection des ancêtres reste pour les Niha nécessaire, car leur conscience religieuse est hantée par l'idée qu'ils vivent dans le péché permanent, qu'ils font figure de "queue de porc» de la communauté chrétienne, du fait de leur marginalité géographique, doublée de leur rattachement tardif à celle-ci et que, pour toutes ces raisons, ils ne profitent guère de la bienveillance divine. Ce sentiment d'abandon exprime dans l'imaginaire leur grande précarité économique et sanitaire, l'isolement et le sous-développement dans lesquels les maintient l'État indonésien, faute d'investissements infrastructurels, et enfin les fortes tensions sociales qui minent leur société de l'intérieur. D'après le tableau qu'en brosse l'auteur, les rivalités entre notables pour l'accès aux fonctions de pouvoir, les contentieux entre familles pour l'appropriation des terres et des arbres fruitiers, ainsi que les dettes de porcs non honorées se conjuguent pour entretenir un climat social délétère au sein des communautés villageoises, chacun suspectant son voisin et même son frère de clan de vouloir le voler ou l'empoisonner.

3 Faire œuvre ethnographique dans ce milieu n'est évidemment pas tâche aisée. En enchaînant sur le mode de la "chronique de terrain ", de courts chapitres qui chaque fois décrivent un contexte d'interaction dans lequel il était directement impliqué, Andrew Beatty restitue de manière très précise et vivante la teneur des rapports sociaux au sein du village d'Orahua où il vécut, mais aussi les multiples obstacles qu'il dut surmonter pour mener à bien sa recherche. La première difficulté à laquelle tout ethnographe est confronté, mais qui prit dans son cas une tournure particulière, est l'acquisition d'une identité et d'un statut qui fassent sens, mais soient aussi honorables pour les gens étudiés. Le chef de village qui l'hébergeait résolut spontanément une partie du problème en attribuant à Andrew Beatty et à son épouse, venue le rejoindre sur place, des teknonymes à consonance locale et en leur assignant des identités claniques complémentaires. Cependant, en contrepartie, le chef demanda à l'ethnologue d'organiser un sacrifice ostentatoire de porcs, nommé owasa, supposé l'inscrire dans le régime d'obligations et de dettes mutuelles au principe du lien social chez les Niha et lui conférer le titre honorifique de « seigneur qui nourrit la terre ». Or, faute d'avoir été épaulé dans les préparatifs et les invitations par les membres de son clan d'adoption, l'ethnologue se ridiculisa en organisant un owasa de piètre qualité et se mit durablement à dos tous ceux qui n'y avaient pas été conviés. Au-delà de cet épisode, le fait d'être identifié à un réseau relationnel particulier dans une société clivée par de multiples rivalités et conflits conduisit Andrew Beatty à dépenser une énergie considérable pour donner des gages de neutralité et ainsi rompre le mur du refus ou du mensonge que lui opposaient la plupart des habitants, ceux-ci le soupçonnant de s'informer pour le compte de leurs ennemis. Dès lors, la réalisation des actes de la recherche les plus élémentaires, tels que le recueil des généalogies - matière à 
controverses entre Niha lors des litiges fonciers -, devenait particulièrement ardue et ne pouvait aboutir qu'à des états partiels et contradictoires. Autre motif de découragement pour l'ethnographe : le fait qu'il ait été quotidiennement exposé à de multiples sollicitations matérielles sans retour - y compris de la part des gens qui lui paraissaient les plus bienveillants - et qu'il devait sans cesse improviser des réponses adaptées aux standards transactionnels locaux afin d'agir avec tact et compassion, sans risquer de se faire intégralement dépouiller. Malgré ces entraves, l'ethnologue put construire avec ses interlocuteurs des échanges intellectuels fructueux, les Niha de son entourage immédiat lui confiant, au fil de conversations dont ils avaient l'initiative, leurs frustrations sociales, leurs songes ou leurs interrogations métaphysiques et lui donnant ainsi accès à des facettes de leur personnalité mais aussi, plus largement, à des aspects du vécu et du ressenti des membres de cette société qui seraient restés secrets autrement.

4 Avec humilité, Andrew Beatty présente After the Ancestors comme le simple récit d'une expérience humaine et des multiples rôles de composition que l'ethnographe doit inventer ou accepter pour gagner l'empathie de ses sujets d'étude. Cependant, ce livre présente un intérêt méthodologique certain, notamment pour les étudiants n'ayant pas encore effectué d'enquête sur le mode de l'observation participante. En restituant fidèlement le verbatim des échanges qu'il eut avec ses interlocuteurs niha dans les contextes d'interaction décrits, l'auteur met admirablement en pratique le style d'écriture dialogique que James Clifford appelait de ses vœux ${ }^{20}$.

\section{NOTES}

20. James Clifford, «Introduction : Partial Truths ", in James Clifford \& George Marcus, eds, Writing Culture. The Poetics and Politics of Ethnography, Berkeley-Los Angeles-London, University of California Press, 1986 : 14-15. 